\title{
Larval zebrafish as a model for glucose metabolism: expression of phosphoenolpyruvate carboxykinase as a marker for exposure to anti-diabetic compounds
}

\author{
B Elo, C M Villano, D Govorko ${ }^{1}$ and L A White \\ Department of Biochemistry and Microbiology, Rutgers, The State University of New Jersey, 76 Lipman Drive, New Brunswick, New Jersey 08901, USA \\ ${ }^{1}$ Biotechnology Center for Agriculture and the Environment, Rutgers, The State University of New Jersey, 59 Dudley Road, New Brunswick, New Jersey 08901, USA \\ (Requests for offprints should be addressed to L A White; Email: lawhite @ aesop.rutgers.edu)
}

\begin{abstract}
The zebrafish model system is one of the most widely used animal models for developmental research and it is now becoming an attractive model for drug discovery and toxicological screening. The completion of sequencing the zebrafish genome and the availability of full-length cDNAs and DNA microarrays for expression analysis, in addition to techniques for generating transgenic lines and targeted mutations, have made the zebrafish model even more attractive to researchers. Recent data indicate that the regulation of glucose metabolism in zebrafish, through the production of insulin, is similar to mammalian models, and many of the genes involved in regulating blood glucose levels have been identified in zebrafish. The data presented here show that adult zebrafish respond to anti-diabetic drugs similarly to mammalian models, by reducing blood glucose levels. Furthermore, we show that the expression of phosphoenolpyruvate carboxykinase (PEPCK), which catalyzes a rate-limiting step in gluconeogenesis and is transcriptionally regulated by glucagon and insulin, is regulated in larval zebrafish similarly to that seen in mammalian systems, and changes in PEPCK expression can be obtained through real-time PCR analysis of whole larval RNA. Taken together, these data suggest that larval zebrafish may be an appropriate model for the examination of glucose metabolism, using PEPCK as an indicator of blood glucose levels.
\end{abstract}

Journal of Molecular Endocrinology (2007) 38, 433-440

\section{Introduction}

The zebrafish model system is becoming one of the most widely used animal models for developmental research because of its fecundity and its genetic and physiological similarities to mammals (Vascotto et al. 1997, Parng et al. 2002). These advantages are leading to the use of the zebrafish model in drug discovery and toxicological screening. The zebrafish embryo is particularly suited to high-throughput screening, due to the small size and optical transparency of the embryo. The utility of the zebrafish as a model organism is further enhanced by the completion of sequencing the zebrafish genome and the availability of full-length cDNAs and DNA microarrays for expression analysis. In addition, techniques for generating transgenic lines, targeted mutation, and nuclear transfer have made the zebrafish model even more useful to researchers.

Hyperglycemia in type II diabetes results from an inability of insulin to control gluconeogenesis (Stumvoll et al. 2005). When glucose is available in the diet, insulin is produced by the pancreas, and gluconeogenesis is inhibited through the downregulation of genes involved in the pathway. In the absence of glucose in the bloodstream, gluconeogenesis is induced by the action of glucagon. Insulin and glucagon are produced in the $\beta$-cells and $\alpha$-cells of the pancreas respectively. As in mammals, the zebrafish pancreas is also comprised of two types of glandular tissues, each of which carries out essential physiological functions (Gnugge et al. 2004). The endocrine tissue is critical for the regulation of glucose metabolism through secretion of insulin, somatostatin, and glucagon directly into the bloodstream. The exocrine portion of the pancreas produces digestive enzymes, including trypsin and amylase, which are carried to the digestive track. Hyperglycemia in type II diabetes has a complex etiology resulting from defects in insulin production and signaling, changes in insulin sensitivity of target tissues, as well as from increased hepatic gluconeogenesis (see Petersen \& Shulman 2006 for review). The genes of zebrafish insulin and glucagon, as well as other important proteins in the regulation of glucose metabolism, have been identified and demonstrate similar regulation patterns and activity as seen in mammalian counterparts (Gnugge et al. 2004, Yee \& Pack 2005, Yee et al. 2005).

Phosphoenolpyruvate carboxykinase (PEPCK) catalyzes the rate-limiting step in gluconeogenesis, i.e. the conversion of oxaloacetate to phosphoenolpyruvate 
(Quinn \& Yeagley 2005). Because PEPCK mRNA expression is transcriptionally modified by several glucoregulatory hormones, including insulin and glucagon, it is an excellent marker to investigate the effects of compounds on the regulation of glucose levels. Glucagon stimulates PEPCK through activation of cyclic AMP (Leahy et al. 1999, Yamada et al. 1999, Herzig et al. 2001, Liu et al. 2001, Monroy et al. 2001, Waltner-Law et al. 2003). In addition, PEPCK gene expression is also induced by glucocorticoids, all-trans retinoic acid, and thyroid hormone (Stafford et al. 2001, Waltner-Law et al. 2003), whereas both glucose and insulin inhibit PEPCK expression (O'Brien et al. 2001).

To examine the potential for zebrafish as a model for the regulation of glucose levels, we exposed adult and larval zebrafish to known anti-diabetic compounds. Our data show that blood glucose levels of the adult zebrafish are reduced following exposure to glipizide, an antidiabetic drug. Furthermore, our data show that PEPCK expression in the larval zebrafish is regulated similarly to that of mammalian systems, with the expression being enhanced by cAMP/dexamethasone and all-trans retinoid acid and significantly reduced by exposure to antidiabetic drugs, glipizide, metformin, and rosiglitazone.

\section{Materials and methods}

\section{Fish maintenance}

The zebrafish ( $\mathrm{AB}$ strain) were maintained in a recirculating system (Aquatic Habitats) in a cycle of $14 \mathrm{~h}$ light:10 h darkness. Embryos were collected at $3 \mathrm{~h}$ post-fertilization (hpf) for experiments.

\section{Exposure of adult zebrafish}

Adult zebrafish were treated transdermally with $25 \%$ glucose in $\mathrm{Me}_{2} \mathrm{SO}$ with or without $1.5 \mathrm{mg} / \mathrm{kg}$ glipizide (Sigma-Aldrich) dissolved in $\mathrm{Me}_{2} \mathrm{SO}$. Blood was collected 5,16 , and $24 \mathrm{~h}$ post-treatment for the analysis of blood glucose levels. Blood glucose levels were measured with OneTouch Ultra (Johnson \& Johnson, New Brunswick, NJ, USA) blood glucosimeter.

\section{Exposure of larval zebrafish}

Zebrafish embryos were collected and allowed to develop until $96 \mathrm{~h}$ post-fertilization (hpf). This age was selected as the yolk sac is diminishing which simulates a starved state. At this time, the collected embryos had either hatched naturally or were mechanically dechorionated to allow for efficient transdermal exposure. The dechorionated embryos were exposed to the selected compounds for $48 \mathrm{~h}$ at $28-30^{\circ} \mathrm{C}$. Treatment concentrations were as follows: cyclic AMP (cAMP: $100 \mu \mathrm{M}$;
Sigma), dexamethasone (DEX: 250, 500, $1000 \mathrm{nM}$; Sigma), glucose (GLU: $40 \mathrm{mM}$; Sigma), metformin (MET: $10 \mu \mathrm{M}$; Sigma), glipizide (GLIP: 100-250 $\mu \mathrm{M}$; Sigma), all-trans retinoic acid (atRA: $10 \mathrm{mM}$; Sigma), and rosiglitazone (ROSI: $1 \mu \mathrm{M}$; Sigma).

\section{RT-PCR}

Embryos collected for RNA isolation were snap frozen and total RNA isolated using TRIzol (Invitrogen) according to the manufacturer's instructions. The isolated RNA was DNase treated (DNA-free kit, Ambion, Austin, TX, USA) to remove genomic DNA contamination and cDNA prepared using the iScript cDNA Synthesis Kit (Bio-Rad). For fluorescent detection of PCR products, reactions containing template and specific primers were amplified using the iCyclerQ Sybr mix (Bio-Rad). Fluorescent PCR products were detected using an iCyclerQ real-time PCR detection system (Bio-Rad). Data were quantified using standard curves generated for each primer set and normalized to $\beta$-actin to control for total RNA concentration. Melting curves were generated for each sample after amplification for the determination of primer dimers, and product amplification was verified using acrylamide gel electrophoresis.

The primer sets are as follows: zf PEPCK forward 5'-GAGAATTCTCACACACAC ACACGTGAGCAGTA-3', reverse $5^{\prime}$-GTAAAAGCTTTCCGCCATAACATCTCCAGC A GAA-3'; zf preproinsulin (insa) forward $5^{\prime}$-AGTGT AAGCACTAACCCAGGCACA- $3^{\prime}$, reverse $5^{\prime}$-TGCAAAGT CAGCCACCTCAGTTTC-3' ${ }^{\prime}$; zf $\beta$-actin forward $5^{\prime}$-CGAG CAGGAGATGG GAACC-3' ${ }^{\prime}$, reverse- $5^{\prime}$ CAACGGAAACG CTCATTGC-3' (Keegan et al. 2002).

\section{Statistical analysis}

Statistical analysis was performed using the SigmaStat v.1.0 computer software package (Systat Software, San Jose, CA, USA). The probability level for statistical significance was $P<0 \cdot 05$. Change in mRNA level was evaluated by one-way ANOVA and either Dunnett's test or Student-Newman-Keuls test was utilized as the multiple comparison method.

\section{Results}

\section{Exposure of adult zebrafish to anti-diabetic compounds reduces blood glucose levels}

There are various classes of anti-diabetic drugs, with different mechanisms of action (Fig. 1). Glipizide is a sulfonylurea, an insulin secretagogue that alters the bond equilibrium between insulin and $\beta$-cells of the pancreas and stimulates endogenous insulin release 


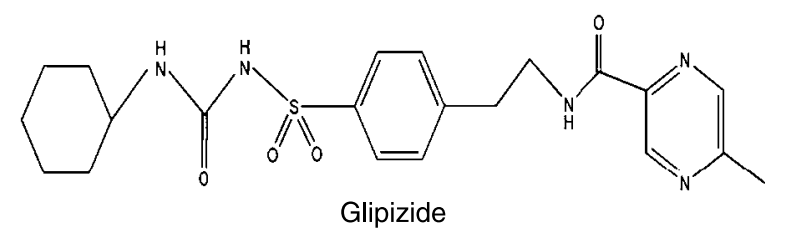<smiles>CC(C)C(=N)NC(=N)N</smiles><smiles>CN(CCOc1ccc(CC2SC(=O)NC2=O)cc1)c1ccccn1</smiles>

Figure 1 Structures of the anti-diabetic compounds used in the experiments.

(Rendell 2004). Metformin is a biguanide that reduces hepatic glucose production by facilitating glucose transporter activity into cells (Goodarzi \& Bryer-Ash 2005). A third mechanism of anti-diabetic action is to sensitize the cell to insulin, such as the action of the thiazolidinediones (i.e. rosiglita zone (ROSI)), which bind to peroxisome proliferatoractivated receptors and induces the transcription of metabolic enzymes (Pietruck et al. 2005).

Two methods of delivering glucose to adult zebrafish were used to determine the most efficient way to elevate blood glucose levels. Adult males were fasted for $48 \mathrm{~h}$ and either fed high-glucose flake food or transdermally exposed to glucose in $\mathrm{Me}_{2} \mathrm{SO}$ by applying it to the abdomen of lightly anesthetized fish. Blood was collected from the zebrafish tail vein and glucose levels determined using a glucosimeter. Feeding of glucose did not significantly alter the blood glucose levels (Fig. 2A). However, transdermal absorption of the glucose effectively elevated the blood glucose. Therefore, all exposures in later experiments using the adult zebrafish were performed using transdermal exposure.

In order to determine whether known anti-diabetic drugs alter the zebrafish blood glucose levels, blood was
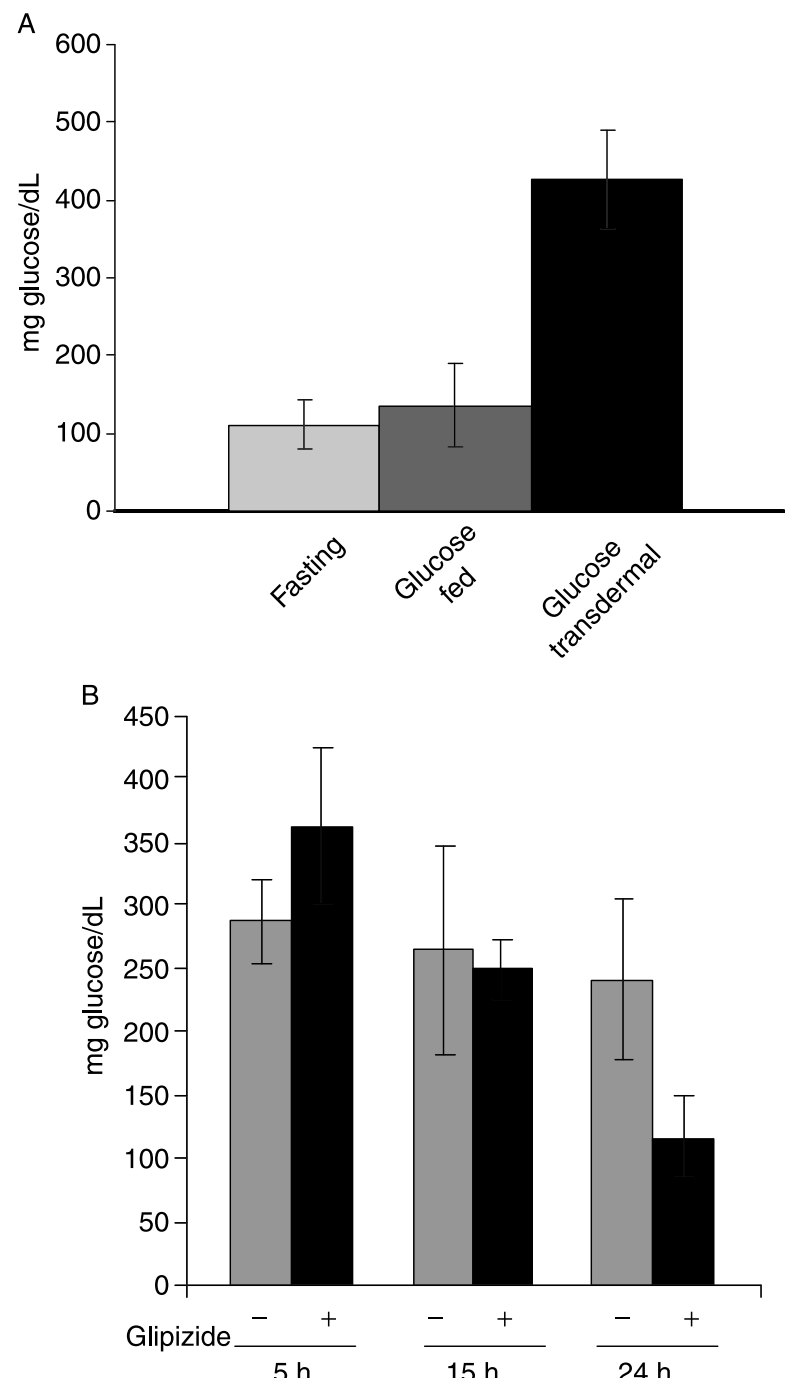

Figure 2 Effects on blood glucose levels in adult zebrafish. (A) Adult male zebrafish were fasted for $48 \mathrm{~h}$ and either fed glucose-rich flakes or exposed to a $25 \%$ glucose/ $/ \mathrm{Me}_{2} \mathrm{SO}$ solution transdermally. Blood glucose was measured 45 min posttreatment using a OneTouch Ultra (Johnson \& Johnson) blood glucosimeter. A minimum of six male zebrafish were used per treatment. (B) Adult male zebrafish were exposed to a $25 \%$ glucose/ $\mathrm{Me}_{2} \mathrm{SO}$ solution transdermally with or without glipizide $(1.5 \mathrm{mg} / \mathrm{ml})$. Blood glucose levels were measured at 5,15 , and $24 \mathrm{~h}$ post-treatment.

collected from adult zebrafish that had been either loaded with glucose alone or co-treated with glucose and glipizide, an FDA-approved anti-diabetic drug. When zebrafish were co-exposed to glucose and glipizide, the blood glucose levels were significantly reduced in comparison with glucose alone by $24 \mathrm{~h}$ postexposure (Fig. 2B). These data suggest that the regulation of blood glucose levels in zebrafish is similar to that observed in mammalian systems. 


\section{PEPCK expression is reduced by exposure of larval zebrafish to exogenous glucose}

To develop the zebrafish as an appropriate model system for screening of compounds that alter glucose metabolism, we decided to use the larval zebrafish. Organ development, including the liver and pancreas, is completed by day 4 (96 hpf) of zebrafish development. Because larval zebrafish do not have the amount of blood necessary for accurate analysis of blood glucose levels, we opted to analyze the expression of PEPCK, which is transcriptionally regulated by glucagon and insulin and is therefore a sensitive marker of blood glucose levels (Yeagley et al. 2000, Cassuto et al. 2005, Chakravarty et al. 2005, Yeagley \& Quinn 2005).

The expression of PEPCK in larval zebrafish was examined using real-time RT-PCR. Total RNA was isolated from larval zebrafish exposed to vehicle or glucose, and the expression of PEPCK was analyzed 24-48 $\mathrm{h}$ post-treatment. The data show that expression of PEPCK was detectable in larval zebrafish, and that exogenous glucose significantly inhibited its expression at both 24 and $48 \mathrm{~h}$ post-exposure (Fig. 3A). These data indicate that PEPCK is a sensitive marker for blood glucose levels in larval zebrafish.

The expression of zebrafish preproinsulin was also examined following exposure to exogenous glucose. Although there are two zebrafish insulin genes, insa and $i n s b$, the expression of insb appears to be restricted to early times in embryonic development (Papasani et al. 2004). Therefore, we focused on the expression of insa, which is expressed in the larval zebrafish. The data show that preproinsulin expression was upregulated by exposure to glucose at both the 24- and 48-h time points (Fig. 3B). These data indicate that the larval zebrafish is absorbing the exogenous glucose and is responding to the increased blood glucose levels as expected.

\section{PEPCK expression is induced by CAMP + dexametha- sone and retinoic acid}

PEPCK is primarily regulated at the level of transcription. Glucagon activates the expression of PEPCK through the activation of cAMP leading to increased binding to cAMP elements in the PEPCK promoter (Leahy et al. 1999, Yamada et al. 1999, Herzig et al. 2001, Liu et al. 2001, Monroy et al. 2001, Waltner-Law et al. 2003). In addition, glucocorticoids, atRA, and thyroid hormone activate PEPCK gene expression (Stafford et al. 2001, Waltner-Law et al. 2003). Therefore, to demonstrate activation of PEPCK expression in the larval zebrafish, we exposed the embryos to cAMP and the glucocorticoid DEX (Fig. 4A). Exposure of zebrafish to cAMP and DEX increased PEPCK mRNA levels $48 \mathrm{~h}$ post-exposure. DEX increased PEPCK in a dose-dependent manner. Next,
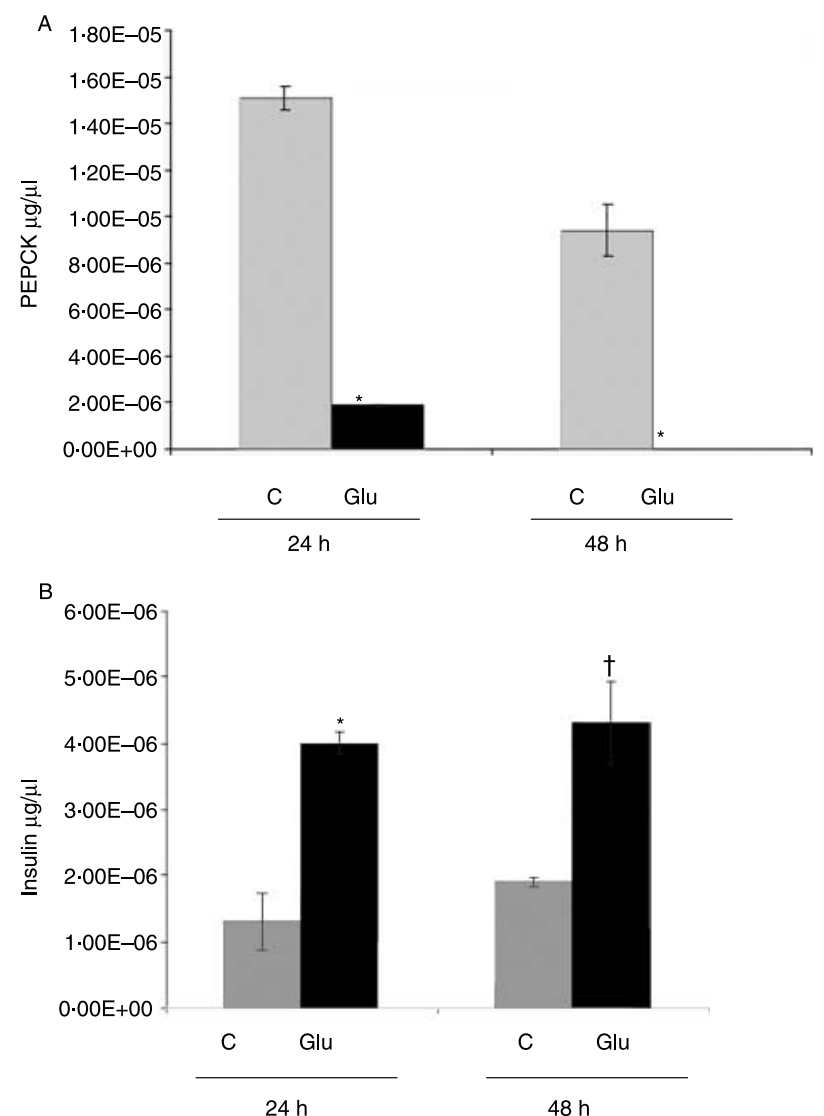

Figure 3 Expression of PEPCK is reduced following exposure of larval (96 hpf) zebrafish. Total RNA isolated from larval zebrafish exposed to $40 \mathrm{mM}$ glucose (GLU) for 24 and $48 \mathrm{~h}$ was used as a template for quantitative RT-PCR using primers specific for zfPEPCK (A), zf-preproinsulin (B), and $\beta$-actin. PCR products were detected using SYBR green as a fluorogenic marker and an icyclerq (Bio-Rad). Data were quantified using a standard curve generated using specific PCR products at concentrations ranging from $100 \mathrm{ng}$ to $1 \mathrm{pg}$. Data were normalized to $\beta$-actin to control for total RNA concentration. Data were plotted as concentration of template after normalization to total RNA concentration by $\beta$-actin. Error bars denote s.D. Statistical significance between control and untreated expression, determined by Student's $t$-test, is denoted by ${ }^{\star} P \leq 0.005$ or $^{\dagger} P \leq 0.02$.

larval zebrafish were exposed to atRA for $48 \mathrm{~h}$, and PEPCK expression was determined using RT-PCR. Data show that exposure to atRA results in a twofold increase in the PEPCK expression (Fig. 4B). These data demonstrate that activation of PEPCK expression in larval zebrafish is regulated similarly to PEPCK in mammalian models.

\section{Inhibition of PEPCK expression by known anti- diabetic compounds}

To investigate the effect of anti-diabetic drugs, larval zebrafish were exposed to cAMP+DEX to elevate 

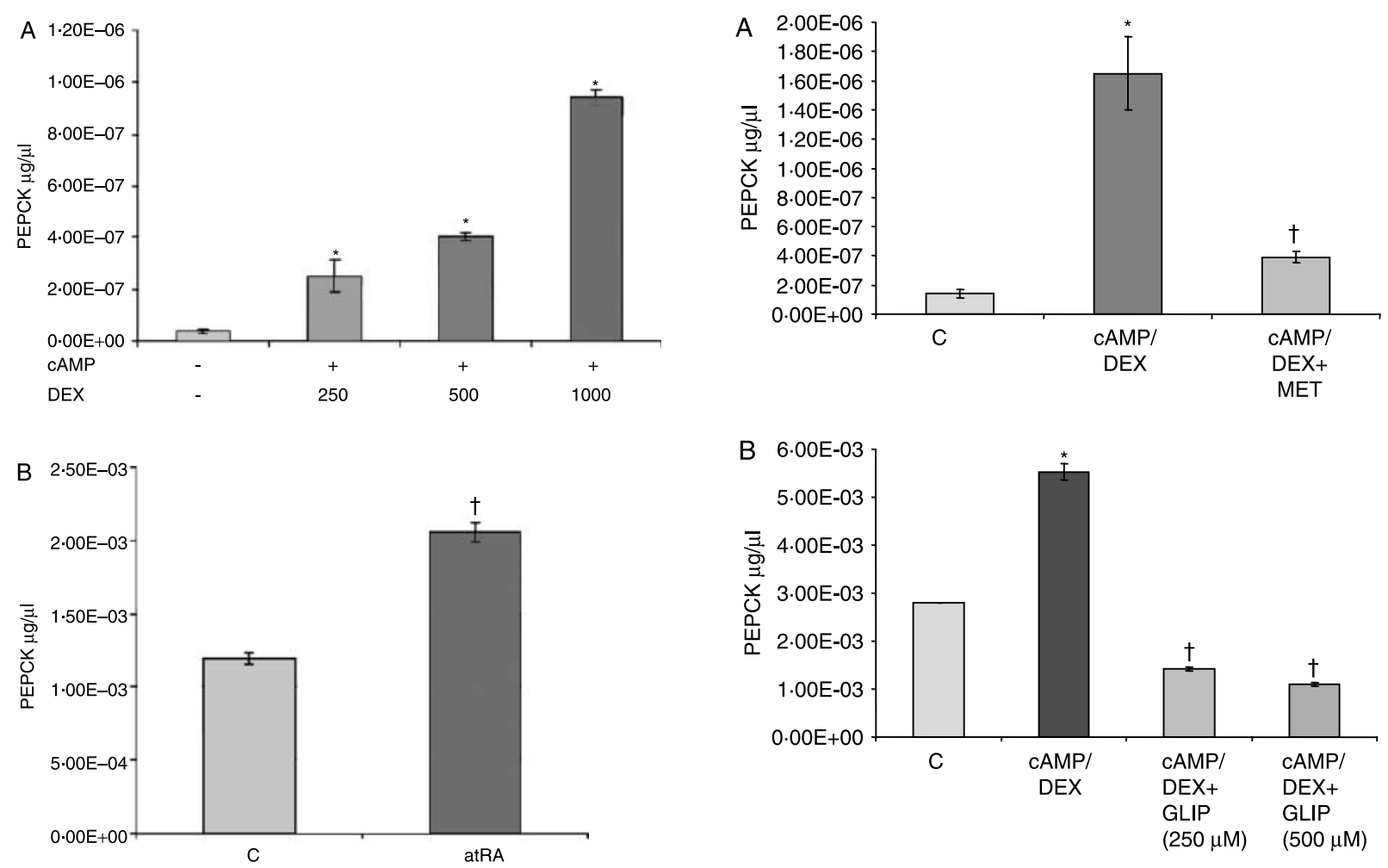

Figure 4 PEPCK expression is enhanced following exposure to cAMP + DEX or atRA. (A) Total RNA isolated from larval zebrafish exposed to cAMP $(100 \mu \mathrm{M})$ and DEX $(250,500$, and $1000 \mathrm{nM})$ for $48 \mathrm{~h}$ was used as a template for quantitative RT-PCR using primers specific for zfPEPCK and $\beta$-actin as described in Fig. 3. Error bars denote S.D. Statistical significance, determined by Student's $t$-test, is denoted by ${ }^{*} P<0 \cdot 005$. (B) Total RNA isolated from larval zebrafish exposed to atRA (10 $\mathrm{nM})$ for $48 \mathrm{~h}$ was used as a template for quantitative RT-PCR as described in (A). Statistical significance, as determined by Student's $t$-test, is denoted by ${ }^{\dagger} P<0.001$.

PEPCK mRNA levels, and co-treated with either MET, GLIP, or ROSI. Co-treatment with metformin resulted in an inhibition of cAMP + DEX activation of PEPCK expression by approximately $75 \%$ (Fig. 5B). Similarly, exposure to glipizide reduced PEPCK expression by $75-80 \%$ (Fig. 5A).

The final anti-diabetic compound tested, rosiglitazone, functions by enhancing insulin sensitivity of extrahepatic tissues (Pietruck et al. 2005). Our data show that exposure of larval zebrafish to rosiglitazone alone results in a slight but significant decrease in PEPCK expression (Fig. 5C). Furthermore, co-exposure with rosiglitazone and cAMP/DEX results in a slight but significant decrease in cAMP + DEX-induced PEPCK expression (Fig. 5C). Taken together, these data indicate that PEPCK expression is a sensitive indicator of blood glucose levels in larval zebrafish and demonstrate that PEPCK regulation in zebrafish is similar to that seen in humans and mice.

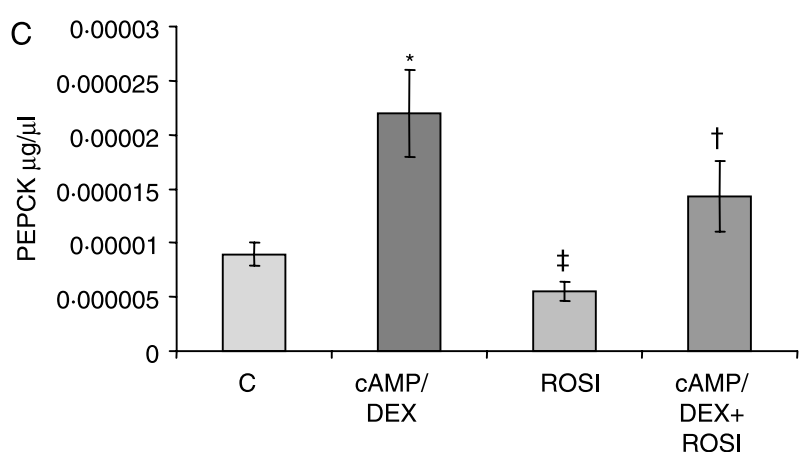

Figure 5 Anti-diabetic compounds reduce PEPCK expression in larval zebrafish. Total RNA isolated from larval zebrafish (96 hpf) exposed to CAMP + DEX or CAMP + DEX and (A) metformin (MET: $10 \mu \mathrm{M}),(\mathrm{B})$ glipizide (GLIP: 250 or $100 \mu \mathrm{M}),(\mathrm{C}) \mathrm{cAMP}+\mathrm{DEX}+$ rosiglitazone $(1 \mu \mathrm{M})$ or rosiglitazone (ROSI) alone for $48 \mathrm{~h}$ and was used as a template for quantitative RT-PCR using primers specific for zfPEPCK and $\beta$-actin as described in Fig. 3. Error bars denote S.D. Statistical significance, determined by Student's $t$-test, is denoted by ${ }^{\star} P<0.001 ;{ }^{\dagger} P \leq 0.005 ;{ }^{\ddagger} P<0.0001$.

\section{Discussion}

The zebrafish is rapidly becoming one of the most widely used animal models for pharmacological and toxicological testing of new compounds. The data presented in this paper indicate that both the adult and larval zebrafish are appropriate models to test the effects of compounds on glucose mobility and 
metabolism. Adult zebrafish blood samples demonstrated that the FDA-approved anti-diabetic drug, glipizide, lowers blood glucose levels in this model system. Data also demonstrate that these compounds act similarly in the larval zebrafish, through examination of PEPCK expression.

The larval zebrafish (96 hpf) is ideal for these studies, since hundreds of embryos can be analyzed simultaneously and organ development has been completed. The zebrafish pancreas, like that of mammalian species, results from the fusion of two buds (ventral anterior and dorsal posterior) emerging from the gut tube at the contact point of the endoderm and the vasculature (Field et al. 2003). The posterior bud gives rise to the endocrine portion of the tissue, whereas the anterior bud provides the exocrine function. As early as $34 \mathrm{hpf}$, the posterior bud is present as a cluster of cells that are already expressing endocrine specific genes. By $52 \mathrm{hpf}$ the anterior and posterior buds have fused, with the anterior bud connecting to the alimentary canal and the posterior bud forming the islet. At $76 \mathrm{hpf}$, the exocrine pancreatic tail is observed, and the islet is completely engulfed by the exocrine tissue. Specific genes are critical for the proper development of the pancreas and are conserved between mammalian and zebrafish model systems. These include one of the earliest markers of pancreatic development, the pancreatic and duodenal homeobox-1 $(p d x-1)$ gene (Milewski et al. 1998). Knockdown of $p d x-1$ in zebrafish results in a reduction of both endocrine and exocrine tissues, with reduced numbers of exocrine cell types, as well as insulin-expressing endocrine cells. Another pathway of interest is the hedgehog signaling pathway (Hh). Sonic-youuo (syu)/sonic hh $(s h h)$ mutant embryos have reduced expression of $p d x-1$, in addition to reduced levels of the endocrine-specific markers, insulin and glucagon (Roy et al. 2001). However, no apparent reduction in exocrine-specific gene expression is observed, indicating that the $s h$ pathway is specific for the development of the endocrine tissue. These data indicate that pancreatic development in zebrafish is regulated by similar pathways as in mammalian model systems.

Two insulin genes have been identified in zebrafish (insa and insb; Papasani et al. 2004, Fostel et al. 2005). This is hypothesized to be the result of a genome duplication event that occurred prior to the divergence of teleost fish. In most cases of gene duplication, the duplicated gene is thought to become a non-functional pseudogene because there is no selective advantage to maintain them (Force et al. 1999). However, in some cases, the duplicated genes are functionally maintained if they either acquire a new function or the duplicated gene maintains only certain specialized functions of the primary gene. In the case of the two insulin genes in zebrafish, both genes have maintained functionality; however, their expression patterns differ. Both insa and insb contain the proteolytic processing sites to convert the proinsulin to insulin (Irwin 2004), and these data indicate that the insulins are processed similarly to mammalian insulin and that the processed zebrafish insulins have a three-dimensional structure that is similar to the mammalian protein (Irwin 2004). By analysis of developmental expression patterns, it is believed that insa functions much like the mammalian homologue in glucose regulation, whereas the insb is believed to play a more important role in development (Papasani et al. 2004).

To investigate the effects of select anti-diabetic compounds in larval zebrafish, we chose to examine the expression of PEPCK. PEPCK catalyzes the first committed step in gluconeogenesis, the conversion of oxaloacetate to phosphoenolpyruvate. Although acute hyperglycemia suppresses the expression of genes involved in gluconeogenesis, including PEPCK, chronic hyperglycemia is associated with increased hepatic glucose production and increased PEPCK expression (Quinn \& Yeagley 2005). The ability of a compound to alter PEPCK expression has been used to determine the efficacy of anti-diabetic compounds in mammalian cell culture models. Furthermore, chronic hyperglycemia, like that observed in type II diabetes, results in increased PEPCK expression and activation of gluconeogenesis. The ability of compounds to downregulate PEPCK is an important aspect of their function as antidiabetic drugs.

The mammalian PEPCK promoter region contains cis-acting elements that mediate activation by glucocorticoids, glucagon, thyroid hormone, and retinoic acid (Quinn \& Granner 1990, Hall et al. 2000, Monroy et al. 2001, Waltner-Law et al. 2003). It has been determined that the proximal promoter $(-477$ to $-1 \mathrm{bp}$ ) contains the sequences required for positive regulation of PEPCK, and that this region also contains sequences that are necessary for glucose-mediated inhibition (Cournarie et al. 1999). Additional elements upstream contribute to the magnitude of the transcriptional activation by glucocorticoids; however, they are not necessary for activation. Work from some laboratories indicate that insulin inhibition of PEPCK does not appear to require specific insulin responsive elements, but instead works by inhibiting activation of the gene through blocking binding to the activation elements. However, a PEPCK-like motif in the promoter is implicated in insulin inhibition (O'Brien et al. 2001).

The entire zebrafish genome has been sequenced and is available online (National Center for Biotechnology Information (NCBI)). The zebrafish PEPCK gene is located on chromosome 17, and approximately $1.2 \mathrm{~kb}$ of the $5^{\prime}$ regulatory sequence is available. Analysis of the potential zfPEPCK promoter using a transcription factor database (MatInspector) revealed several elements that are also found in the 
human promoter, including the cAMP-binding sites and CAAT enhancer, which mediate activation by glucagon, and several AP-1 elements and serum response elements, as well as elements involved in tissue and developmental specific regulation (PDX, PAX; Ghosh 1991, 1992). The zebrafish PEPCK promoter also contains several hepatic nuclear factor $3 \beta$ elements, and several PEPCK-like motifs are also present in this $1.2 \mathrm{~kb}$ sequence.

The similarity between the mammalian and zebrafish PEPCK promoters suggests that the zfPEPCK promoter contains the regulatory elements necessary for proper tissue-specific expression, as well as expression in response to glucagons, glucocorticoids, and insulin. The data presented in this paper confirm the similarity in the regulation of the zebrafish and mammalian PEPCK genes. The cAMP and the glucocorticoid dexamethasone activate PEPCK expression, as does exposure to atRA. Furthermore, acute hyperglycemia caused a significant reduction in PEPCK expression.

The anti-diabetic compounds used in this study all regulate blood glucose levels through distinct mechanisms. Glipizide and related sulfonylureas stimulate insulin release, in addition to affecting glucose and insulin sensitivity in extrahepatic tissues (Rendell 2004). Metformin inhibits the endogenous production and release of non-carbohydrate-derived glucose (Goodarzi \& Bryer-Ash 2005). Rosiglitazone is a thiazolidinedione, which binds to the peroxisome proliferator-activated receptors (PPARs), and functions as an anti-diabetic compound by enhancing the sensitivity of the peripheral tissues to insulin (Pietruck et al. 2005). Our data also show that the FDA-approved anti-diabetic drugs metformin, glipizide, and rosiglitazone reduce the expression of zfPEPCK. Given that chronic hyperglycemia activates PEPCK expression and ultimately gluconeogenesis, one critical function of these compounds is to reduce PEPCK expression. Our data in zebrafish is similar to that seen in mammalian tissue culture. For example, sulfonylurea and metformin exposure reduce PEPCK expression in liver cell lines and rat hepatocytes respectively(Davies et al. 1999, Yuan et al. 2002). Rosiglitazone can directly affect PEPCK expression, via PPAR elements in the promoter region (Duplus et al. 2003). However, data from hepatocytes indicate that rosiglitazone is not effective in regulating the PEPCK expression in this model (Davies et al. 2001), causing only a slight decrease. This is similar to our data showing that in the larval zebrafish, exposure to rosiglitazone results in a slight but significant decrease in PEPCK expression.

In summary, our data demonstrate that regulation of PEPCK in larval zebrafish mimics that seen in mammalian cells. This is further supported by the data from other laboratories demonstrating that the ortholog to the human glucose transporter GLUT1 has been identified in zebrafish (Jensen $e$ al. 2006). These findings suggest that the larval zebrafish may be an ideal model in which to examine the effects of novel compounds on glucose metabolism and transport.

\section{Acknowledgements}

We wish to thank J Hillegass for help with zebrafish husbandry and Jessie Maguire for help with manuscript figures. We would also like to thank Drs Ilya Raskin and David Ribnicky for their helpful discussions and comments. The authors declare that there is no conflict of interest that would prejudice the impartiality of this scientific work.

\section{Funding}

This work was supported by the NIH Center for Dietary Supplements Research on Botanicals and Metabolic Syndrome, grant no. 1-P50 AT002776-01, and Rutgers University (L A White) and by the G H Cook Honors Program (B Elo).

\section{References}

Cassuto H, Kochan K, Chakravarty K, Cohen H, Blum B, Olswang Y, Hakimi P, Xu C, Massillon D, Hanson RW et al. 2005 Glucocorticoids regulate transcription of the gene for phosphoenolpyruvate carboxykinase in the liver via an extended glucocorticoid regulatory unit. Journal of Biological Chemistry 280 33873-33884.

Chakravarty K, Cassuto H, Reshef L \& Hanson RW 2005 Factors that control the tissue-specific transcription of the gene for phosphoenolpyruvate carboxykinase-C. Critical Reviews in Biochemistry and Molecular Biology 40 129-154.

Cournarie F, Azzout-Marniche D, Foretz M, Guichard C, Ferre P \& Foufelle F 1999 The inhibitory effect of glucose on phosphoenolpyruvate carboxykinase gene expression in cultured hepatocytes is transcriptional and requires glucose metabolism. FEBS Letters 460 $527-532$.

Davies GF, Khandelwal RL \& Roesler WJ 1999 Troglitazone inhibits expression of the phosphoenolpyruvate carboxykinase gene by an insulin-independent mechanism. Biochimica et Biophysica Acta 1451 $122-131$.

Davies GF, Khandelwal RL, Wu L, Juurlink BH \& Roesler WJ 2001 Inhibition of phosphoenolpyruvate carboxykinase (PEPCK) gene expression by troglitazone: a peroxisome proliferator-activated receptor-gamma (PPARgamma)-independent, antioxidant-related mechanism. Biochemical Pharmacology 62 1071-1079.

Duplus E, Benelli C, Reis AF, Fouque F, Velho G \& Forest C 2003 Expression of phosphoenolpyruvate carboxykinase gene in human adipose tissue: induction by rosiglitazone and genetic analyses of the adipocyte-specific region of the promoter in type 2 diabetes. Biochimie 85 1257-1264.

Field HA, Dong PD, Beis D \& Stainier DY 2003 Formation of the digestive system in zebrafish. II. Pancreas morphogenesis. Developmental Biology 261 197-208.

Force A, Lynch M, Pickett FB, Amores A, Yan YL \& Postlethwait J 1999 Preservation of duplicate genes by complementary, degenerative mutations. Genetics 151 1531-1545. 
Fostel J, Choi D, Zwickl C, Morrison N, Rashid A, Hasan A, Bao W, Richard A, Tong W, Bushel PR et al. 2005 Chemical effects in biological systems-data dictionary (CEBS-DD): a compendium of terms for the capture and integration of biological study design description, conventional phenotypes, and omics data. Toxicological Sciences 88 585-601.

Ghosh D 1991 New developments of a transcription factor database. Trends in Biochemical Science 16 445-447.

Ghosh D 1992 TFD: the transcription factors database. Nucleic Acids Research 20 2091-2093.

Gnugge L, Meyer D \& Driever W 2004 Pancreas development in zebrafish. Methods in Cell Biology 76 531-551.

Goodarzi MO \& Bryer-Ash M 2005 Metformin revisited: re-evaluation of its properties and role in the pharmacopoeia of modern antidiabetic agents. Diabetes, Obesity and Metabolism 7 654-665.

Hall RK, Yamasaki T, Kucera T, Waltner-Law M, O'Brien R \& Granner DK 2000 Regulation of phosphoenolpyruvate carboxykinase and insulin-like growth factor-binding protein-1 gene expression by insulin. The role of winged helix/forkhead proteins. Journal of Biological Chemistry 275 30169-30175.

Herzig S, Long F, Jhala US, Hedrick S, Quinn R, Bauer A, Rudolph D, Schutz G, Yoon C, Puigserver P et al. 2001 CREB regulates hepatic gluconeogenesis through the coactivator PGC-1. Nature 413 179-183.

Irwin DM 2004 A second insulin gene in fish genomes. General and Comparative Endocrinology 135 150-158.

Jensen PJ, Gitlin JD \& Carayannopoulos MO 2006 GLUT1 deficiency links nutrient availability and apoptosis during embryonic development. Journal of Biological Chemistry 281 13382-13387.

Leahy P, Crawford DR, Grossman G, Gronostajski RM \& Hanson RW 1999 CREB binding protein coordinates the function of multiple transcription factors including nuclear factor I to regulate phosphoenolpyruvate carboxykinase (GTP) gene transcription. Journal of Biological Chemistry 274 8813-8822.

Liu X, Wall QT, Taylor L \& Curthoys NP 2001 C/EBPbeta contributes to cAMP-activated transcription of phosphoenolpyruvate carboxykinase in LLC-PK(1)-F + cells. American Journal of Physiology. Renal Physiology 281 F649-F657.

Milewski WM, Duguay SJ, Chan SJ \& Steiner DF 1998 Conservation of PDX-1 structure, function, and expression in zebrafish. Endocrinology 139 1440-1449.

Monroy MA, Ruhl DD, Xu X, Granner DK, Yaciuk P \& Chrivia JC 2001 Regulation of cAMP-responsive element-binding protein-mediated transcription by the SNF2/SWI-related protein, SRCAP. Journal of Biological Chemistry 276 40721-40726.

O'Brien RM, Streeper RS, Ayala JE, Stadelmaier BT \& Hornbuckle LA 2001 Insulin-regulated gene expression. Biochemical Society Transactions 29 552-558.

Papasani MR, Gensure RC, Yan YL, Gunes Y, Postlethwait JH, Ponugoti B, John MR, Juppner H \& Rubin DA 2004 Identification and characterization of the zebrafish and fugu genes encoding tuberoinfundibular peptide 39. Endocrinology 145 5294-5304.

Parng C, Seng WL, Semino C \& McGrath P 2002 Zebrafish: a preclinical model for drug screening. Assay and Drug Development Technology 1 41-48.

Petersen KF \& Shulman GI 2006 Etiology of insulin resistance. American Journal of Medicine 119 S10-S16.

Pietruck F, Kribben A, Van TN, Patschan D, Herget-Rosenthal S, Janssen O, Mann K, Philipp T \& Witzke O 2005 Rosiglitazone is a safe and effective treatment option of new-onset diabetes mellitus after renal transplantation. Transplant International 18 483-486.
Quinn PG \& Granner DK 1990 Cyclic AMP-dependent protein kinase regulates transcription of the phosphoenolpyruvate carboxykinase gene but not binding of nuclear factors to the cyclic AMP regulatory element. Molecular and Cellular Biology 10 3357-3364.

Quinn PG \& Yeagley D 2005 Insulin regulation of PEPCK gene expression: a model for rapid and reversible modulation. Current Drug Targets. Immune, Endocrine and Metabolic Disorders 5 423-437.

Rendell M 2004 The role of sulphonylureas in the management of type 2 diabetes mellitus. Drugs 64 1339-1358.

Roy S, Qiao T, Wolff C \& Ingham PW 2001 Hedgehog signaling pathway is essential for pancreas specification in the zebrafish embryo. Current Biology 11 1358-1363.

Stafford JM, Waltner-Law M \& Granner DK 2001 Role of accessory factors and steroid receptor coactivator 1 in the regulation of phosphoenolpyruvate carboxykinase gene transcription by glucocorticoids. Journal of Biological Chemistry 276 3811-3819.

Stumvoll M, Goldstein BJ \& van Haeften TW 2005 Type 2 diabetes: principles of pathogenesis and therapy. Lancet 365 1333-1346.

Vascotto SG, Beckham Y \& Kelly GM 1997 The zebrafish's swim to fame as an experimental model in biology. Biochemistry and Cell Biology $\mathbf{7 5}$ 479-485.

Waltner-Law M, Duong DT, Daniels MC, Herzog B, Wang XL, Prasad R \& Granner DK 2003 Elements of the glucocorticoid and retinoic acid response units are involved in cAMP-mediated expression of the PEPCK gene. Journal of Biological Chemistry 278 10427-10435.

Yamada K, Duong DT, Scott DK, Wang JC \& Granner DK 1999 CCAAT/enhancer-binding protein beta is an accessory factor for the glucocorticoid response from the cAMP response element in the rat phosphoenolpyruvate carboxykinase gene promoter. Journal of Biological Chemistry 274 5880-5887.

Yeagley D \& Quinn PG 2005 3',5'-Cyclic adenosine monophosphate response element-binding protein and CCAAT enhancer-binding protein are dispensable for insulin inhibition of phosphoenolpyruvate carboxykinase transcription and for its synergistic induction by protein kinase A and glucocorticoids. Molecular Endocrinology 19 913-924.

Yeagley D, Moll J, Vinson CA \& Quinn PG 2000 Characterization of elements mediating regulation of phosphoenolpyruvate carboxykinase gene transcription by protein kinase A and insulin. Identification of a distinct complex formed in cells that mediate insulin inhibition. Journal of Biological Chemistry $\mathbf{2 7 5}$ 17814-17820.

Yee NS \& Pack M 2005 Zebrafish as a model for pancreatic cancer research. Methods in Molecular Medicine 103 273-298.

Yee NS, Lorent K \& Pack M 2005 Exocrine pancreas development in zebrafish. Developmental Biology 282 84-101.

Yuan L, Ziegler R \& Hamann A 2002 Inhibition of phosphoenolpyruvate carboxykinase gene expression by metformin in cultured hepatocytes. Chinese Medical Journal 115 1843-1848.

Received in final form 4 December 2006

Accepted 3 January 2007

Made available online as an Accepted Preprint 24 January 2007 